\title{
Primeiro registro de Atta sexdens rubropilosa Forel, 1908 (Hymenoptera: Formicidae) atacando noni (Morinda Citrifolia L.) em Colorado do Oeste (RO), Brasil
}

\author{
First report of Atta sexdens rubropilosa Forel, 1908 (Hymenoptera: Formicidae) \\ damaging noni (Morinda citrifolia L.) in Colorado do Oeste (RO), Brazil
}

\author{
Willian Daróz Matte ${ }^{\text {* }}$, Lucas Ferraz de Queiroz¹, Janaína de Nadai Corassa

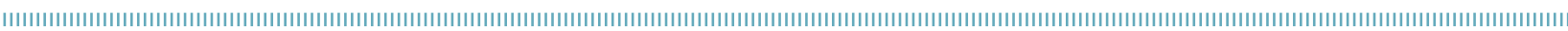

RESUMO: Este trabalho teve como objetivo registrar o ataque de Atta sexdens rubropilosa Forel, 1908 (Hymenoptera, Formicidae) em noni, Morinda citrifolia L. Este registro foi ao acaso. As operárias foram coletadas manualmente em junho de 2015 na região sul de Rondônia, Brasil. O desfolhamento pelas formigas foi registrado em uma planta de noni com quatro anos de idade. No local foi encontrado apenas um sauveiro. A desfolha aconteceu de baixo para cima. O horário de forrageamento concentrava-se no período noturno. Esse é o primeiro registro dessa formiga cortando folhas de noni, no estado de Rondônia e no Brasil.

PALAVRAS-CHAVE: forrageamento; formigas; saúva.
ABSTRACT: This study aimed store cord the attack of Atta sexdens rubropilosa Forel, 1908 (Hymenoptera, Formicidae) in noni, Morinda citrifolia L. This record was random. The workers were collected manually in June 2015 in the southern region of Rondonia, Brazil. The defoliation by ants was recorded in a noniplant four years old. On the site we found only one ant nest. Defoliation happened from the bottom up. The foraging time was concentrated at night. This is the first record of ants cutting leaves of noni in the state of Rondonia and Brazil.

KEYWORDS: foraging; ants; ant. 
A espécie Morinda citrifolia L. é uma planta de pequeno porte, com 3 a $10 \mathrm{~m}$ de altura quando adulta. Pertencente à família Rubiaceae e com origem no sudeste asiático e na Austrália, atualmente o seu cultivo se apresenta bastante distribuído nas regiôes tropicais. O fruto é conhecido popularmente como noni (Nelson, 2006). É uma cultura relativamente recente no Brasil, e nos últimos 10 anos aumentou o interesse pelo seu cultivo comercial por conta de suas propriedades medicinais. A casca tem propriedade adstringente e é utilizada no tratamento contra malária; as folhas são usadas como analgésico e no tratamento de inflamaçôes externas; as flores são empregadas no tratamento de inflamaçóes oculares; as sementes são aplicadas como laxante; e os frutos possuem a mais ampla utilizaçáo, podendo atuar como antibactericida, analgésico, anticongestivo, antioxidante, expectorante, anti-inflamatório, adstringente, emoliente, emenagogo, laxativo, analgésico, hipotensor, purificador do sangue, imunoestimulante e tônico. Também é atribuída ao fruto ação anticancerígena (Rodríguez; Pinedo, 2005 apud Sousa et al., 2010). Não há registro de quando foi introduzida sua cultura no estado de Rondônia, entretanto sabe-se que seu cultivo vem sendo realizado em pequena escala por diversos produtores, mais para o consumo do que para o comércio.

No entanto, como qualquer outra cultura agrícola, o cultivo do noni pode ser limitado pela incidência de insetos praga. Entre eles, citam-se as formigas cortadeiras do gênero Atta e Acromyrmex, principais insetos daninhos às culturas no Brasil por causa da sua grande capacidade de cortar as folhas (Anjos et al., 1993).

A tribo Attini (ordem: Hymenoptera, família: Formicidae, subfamília: Myrmicinae) compreende mais de 230 espécies de formigas divididas em 13 gêneros (Schultz; Brady, 2008). As formigas do gênero Atta são conhecidas popularmente como saúvas ou "formigas-cortadeiras", possuem o hábito de cortar elevadas quantidades de matéria vegetal utilizadas para o cultivo de um fungo mutualista que serve de alimento para as formas larvais e adultas. O micélio, juntamente com o material vegetal, resulta numa estrutura conhecida como “jardim de fungos” (Rodrigues et al., 2008).

Não há, na literatura, referências sobre o ataque de formigas-cortadeiras na cultura do noni. Assim, este trabalho teve por objetivo registrar a ocorrência de saúvas na cultura do noni localizada no estado de Rondônia, Brasil.

A área de incidência caracteriza-se por uma planta de noni com aproximadamente quatro anos e que se situava entre culturas cítricas, situada na zona rural do município de

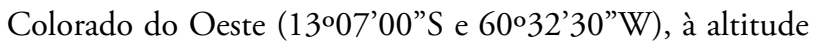
de $460 \mathrm{~m}$ no sul do estado de Rondônia. O noni foi plantado em uma área destinada para horticultura de cerca de 0,5 ha. A vistoria foi realizada no mês de junho de 2015 com a finalidade de se descobrir o que estava atacando a planta noni, pois até o momento nunca se tinha visto praga nenhuma cortar as folhas dessa cultura. $\mathrm{Na}$ ocasião, toda a área foi percorrida para localização e quantificaçáo dos formigueiros e das plantas danificadas. Após constatação da presença de formigas-cortadeiras, observaram-se as atividades de corte e de carregamento das folhas e foram coletados exemplares de forma manual, inseridos em recipientes com ventilação, para posterior determinação da espécie.

As formigas encontradas atacando a planta de noni foram identificadas como pertencentes à espécie Atta sexdens rubropilosa Forel, 1908 (Fig. 1). Esse é o primeiro registro dessa espécie cortando folhas de noni em Rondônia e no restante do Brasil. Essa espécie, conhecida como saúva-limão, é uma formiga-cortadeira de grande importância econômica por forragear em plantas de culturas agrícolas, florestais e pecuárias às mais diversificadas (Mariconi et al., 1963; Kramer; Kozlowski, 2009 apud Souza et al., 2009).

Foi encontrado na área apenas um sauveiro (Fig. 2), localizado numa área de mata nativa, a $20 \mathrm{~m}$ da área do cultivo do noni. Em função da densidade de resíduos vegetais apresentados no local, definidos visualmente, em diversos estágios de decomposição, não foi possível medir o tamanho do formigueiro.

Deparou-se apenas com um olheiro (Fig. 2) cortando as folhas de noni. Para Autuori (1941), o primeiro olheiro de um ninho de $A$. sexdens rubropilosa só é aberto após 87 dias de sua fundação, e o segundo surge 14 meses depois. Assim, é possível que tal sauveiro tivesse menos de um ano de idade.

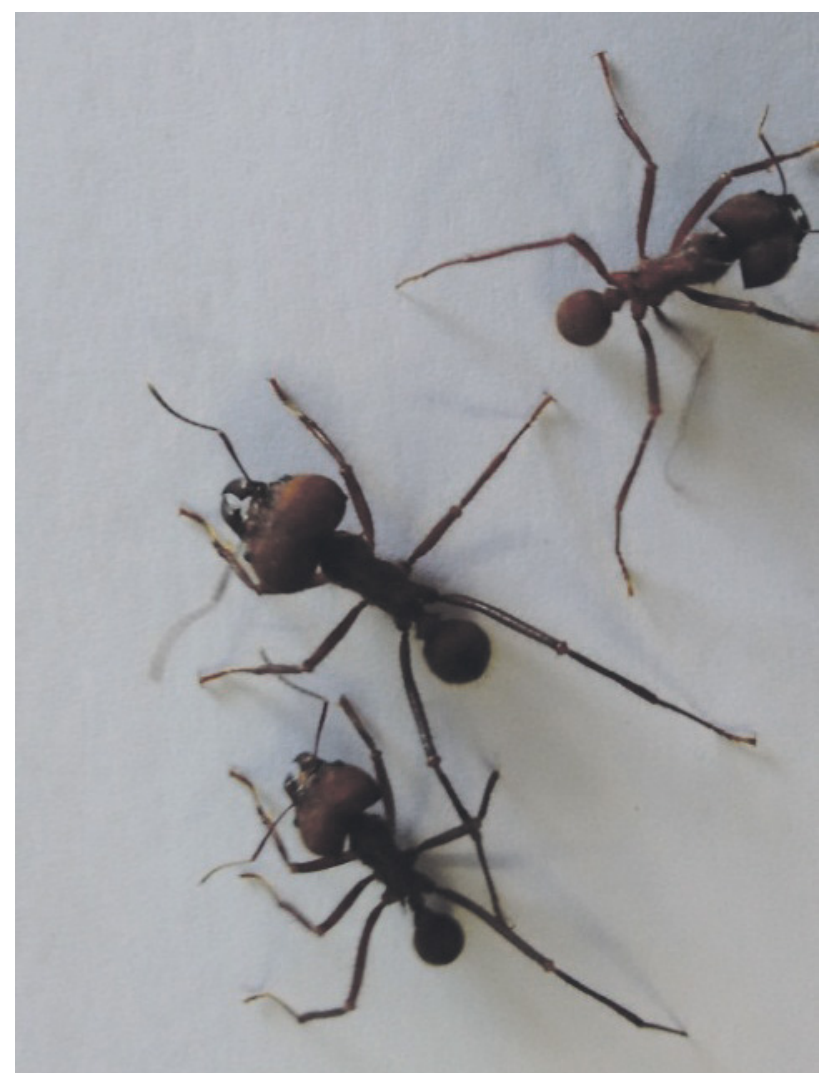

Figura 1. Atta sexdens rubropilosa Forel, 1908 coletada na área. Colorado do Oeste, Rondônia, junho de 2015. 
A planta de noni estava a $20 \mathrm{~m}$ da abertura do único olheiro, que não possuía muita terra solta em volta (Fig. 3). Esse sauveiro estava em atividade (Fig. 4) no momento em que foi encontrado, por volta das $18 \mathrm{~h}$. Os indivíduos coletados tinham coloração mais clara e eram de tamanho pequeno. Não se observou a presença de soldados, o que indica se tratar de um sauveiro novo (AUTUORI, 1941).

Como o ataque estava no início, foi constatada 5\% de desfolha na planta. O horário de forrageamento das formigas iniciava-se por volta do anoitecer, entre $18 \mathrm{~h}$ e $19 \mathrm{~h}$, perpetuando-se pela noite. Essas atividades foram registradas nas datas do dia 15 de junho e confirmadas em 16, 17 e 18 de junho de 2015 (Fig. 5).

Como descrição das atividades de corte, coleta e transporte de folhas pelas operárias do sauveiro, constatou-se que um grupo de saúvas subia na árvore e começava o corte das folhas das extremidades para o centro em formato de meia-lua (hábito peculiar às formigas-cortadeiras). Essas formigas derrubavam as partes cortadas das folhas no chão. Outro grupo

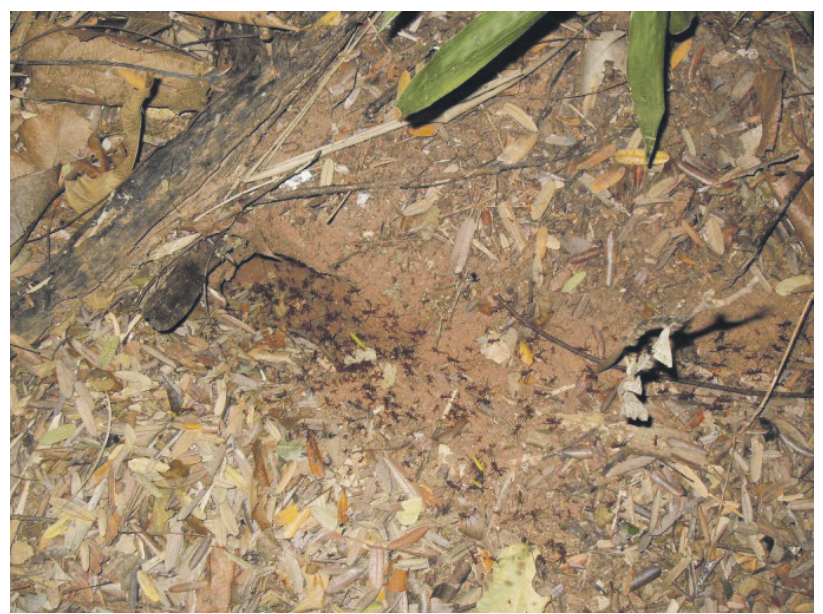

Figura 2. Sauveiro em atividade, Colorado do Oeste, Rondônia, junho de 2015.

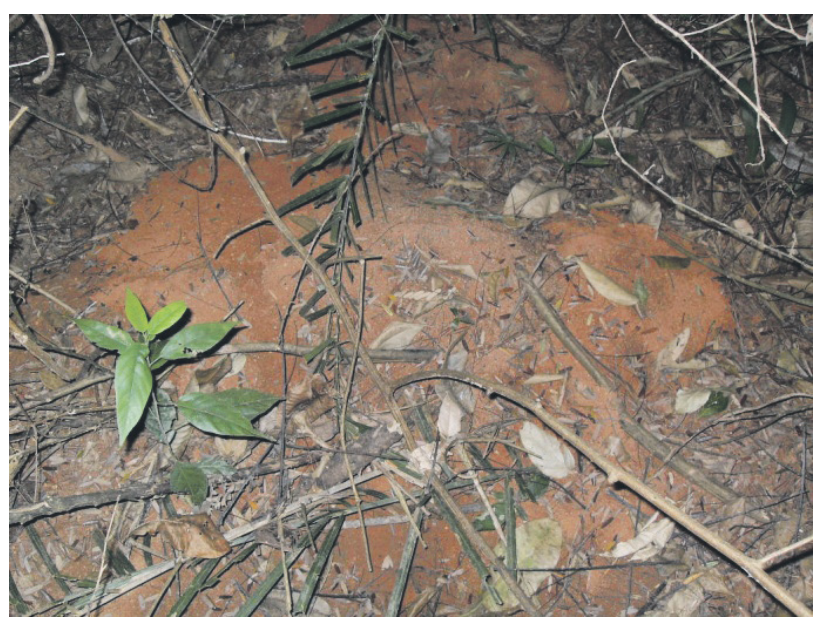

Figura 3. Terra solta resultante da abertura de galerias subterrâneas pelas formigas. Colorado do Oeste, Rondônia, junho de 2015. de saúvas recolhia os cortes e transportava-os para dentro do sauveiro. Souza et al. (2009) perceberam esse mesmo comportamento em $A$. sexdens rubropilosa, ao registrar seu ataque em árvores de nim, Azadirachta indica A. Juss., no estado de Minas Gerais.

Verificou-se ainda que a planta estava sendo desfolhada de baixo para cima, e as folhas no ápice da copa não estavam sendo atacadas. Esse é um padrão de desfolhamento diferente do relatado por ANDRADE (1939 apud Souza et al., 2009) e por Amante (1967), no qual as operárias cortam o material vegetal sempre no sentido de cima para baixo, mas coincide com as conclusóes de Anjos; Cruz (1997) e Souza et al. (2009).

Ataques por formigas-cortadeiras às folhas de Morinda citrifolia não haviam sido registrados no local até o presente momento. Diante do exposto, afirma-se que é possível ocorrer $o$ ataque de formigas-cortadeiras na cultura do noni, podendo ela se tornar uma praga em potencial para a cultura.

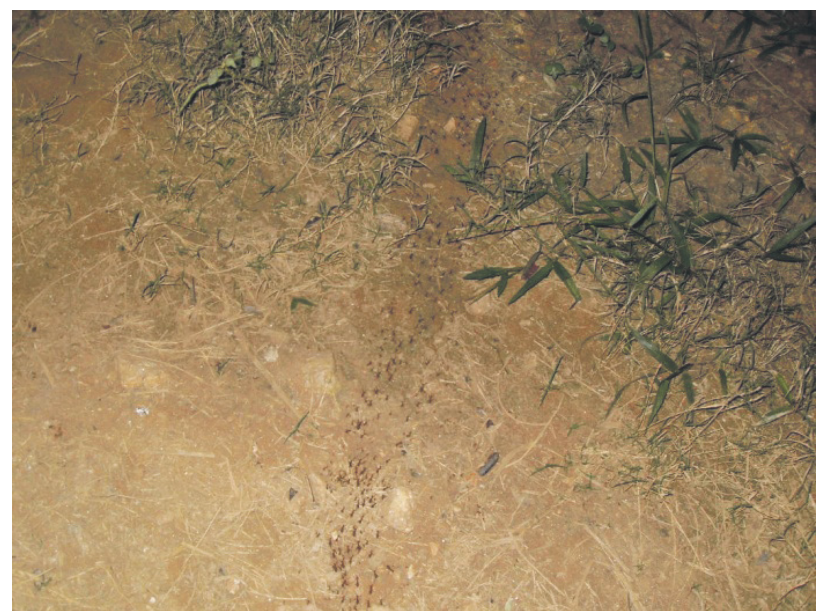

Figura 4. Atividade das formigas-saúvas. Colorado do Oeste, Rondônia, junho de 2015.

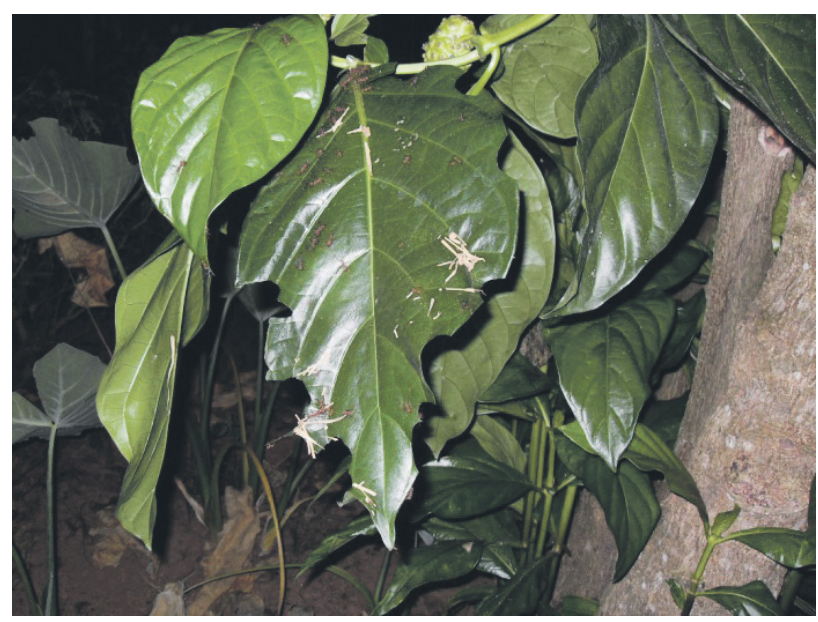

Figura 5. Desfolha ocasionada por formigas-saúvas em planta de noni. Colorado do Oeste, Rondônia, junho de 2015. 


\section{REFERÊNCIAS}

AMANTE, E. Prejuízos causados pela formiga saúva em plantações de Eucalyptus e Pinus no estado de São Paulo. Silvicultura em São Paulo, v.6, p.355-363, 1967.

ANJOS, N.; CRUZ, A.P. Preferência de posição na copa de eucaliptos para o ataque de formigas cortadeiras. In: ENCONTRO DE MIRMECOLOGIA, 13., 1997, Ilhéus, BA. Resumos. Ilhéus, 1997. p. 115.

ANJOS, N.; MOREIRA, D.D.O.; DELLA LUCIA, T.M.C. Manejo integrado de formigas cortadeiras em reflorestamentos. In: DELLA LÚCIA, T.M.C. As formigas cortadeiras. Viçosa: Folha de Viçosa, 1993. p.212-241.

AUTUORI, M. Contribuição para o conhecimento da saúva (Atta spp. Hymenoptera, Formicidae). I- Evolução do sauveiro (Atta sexdens rubropilosa Forel 1908). Arquivos do Instituto Biológico, São Paulo, v.12, n.12, p.197-228, 1941.

MARICONI, F.A.M.; ZAMITH, A.P.L.; CASTRO, U.P.; JOLY, S. Nova contribuição para o conhecimento das saúvas de Piracicaba (Atta spp.) (Hym. Formicidae). Revista de Agricultura, v.38, n.2, p.85-93, 1963.
NELSON, S.C. Species profiles for Pacific Island agroforestry: Morinda citrifolia (noni). Permanent Agriculture Resources, Havaí, 2006. Disponível em: <http://citeseerx.ist.psu.edu/viewdoc/ download?doi=10.1.1.190.10\&rep=rep 1 \&type=pd $>$. Acesso em: 3 jun. 2015.

RODRIGUES, A.; BACCI, M.; MUELLER, U.G.; ORTIZ, A.; PAGNOCCA, F.C. Microfungal weeds in the leafcutter ant symbiosis. Microbial Ecology, New York, v.56, p.614-604, 2008.

SCHULTZ, T.R.; BRADY, S.G. Major evolutionary transitions in ant agriculture. Proceeding of the National Academy of Sciences of the United States of America, Washington, v. 105, n. 14, p.54355440, 2008.

SOUSA, J.A.; AQUINO, A.R.L.; FREIRE, F.C.O.; SILVA NETO, P.A.F. Produção de mudas de noni (Morinda citrifolia L.). Comunicado Técnico 157. Fortaleza, jun. 2010.

SOUZA, R.M.; ANJOS, N.; CORDEIRO, G.; MOURÃO, S.A. Primeiro registro de Atta sexdens rubropilosa Forel atacando árvores de nim, Azadirachta indica A. Juss. Arquivos do Instituto Biológico, São Paulo, v.76, n.4, p.729-733, out./dez. 2009. 